\title{
Perspective: autologous skeletal muscle cells for the treatment of fecal incontinence
}

\author{
J. M. Gimble ${ }^{1,2}$
}

Received: 16 August 2015/Accepted: 16 August 2015/Published online: 23 September 2015

(C) Springer-Verlag Italia Srl 2015

Fecal incontinence afflicts a substantial percentage of the population and increases in frequency with advancing age. While physicians and surgeons have developed an array of noninvasive and surgical approaches to this disorder, for many patients it remains a chronic condition that has considerable impact on quality of life and socialization. Consequently, investigators have continued to seek out novel therapeutic approaches. In the current issue of Techniques in Coloproctology, Romaniszyn et al. [1] describe a regenerative surgical approach to fecal incontinence. They designed their study based in part on (a) a preclinical rat model reporting that the injection of bone marrow-derived mesenchymal stem cells (MSC), also known as multipotent stromal cells [2], (b) quantitatively improved objective measures of fecal incontinence [3, 4]; and (c) a pilot clinical study by Frudinger et al. [5] reporting that women with fecal incontinence showed objective improvement in rectal function for up to 1 year following injection of skeletal muscle-derived cells. Here, Romaniszyn et al. [1] report positive outcomes in a pilot study treating fecal incontinence with autologous skeletal muscle cell injection. The authors enrolled ten patients (nine females and one male) with severe fecal incontinence that had persisted despite 6 months of conventional biofeedback therapy. Each patient underwent harvesting of $1 \mathrm{cc}$ of skeletal muscle which was used to isolate and

J. M. Gimble

jgimble@tulane.edu

1 Center for Stem Cell Research and Regenerative Medicine and the Departments of Medicine, Structural and Cellular Biology, and Surgery, Tulane University School of Medicine, New Orleans, LA, USA

2 LaCell LLC, New Orleans, LA, USA culture expand autologous skeletal muscle cells over a 5- to 6-week period. At a subsequent visit, the patient's own skeletal muscle cells were injected into the rectal sphincter with ultrasound guidance. The patients were followed for up to 12 months using quantitative and qualitative outcome measures. One of the ten patients was lost to follow up during the study period. By 18 weeks of follow-up, twothirds of the patients $(n=6)$ reported subjective improvement in symptoms and over half had improved quantitative parameters of rectal function; however, after 1 year of follow-up, two of these patients exhibited a decline in both their qualitative and quantitative improvements [1]. This report by Romaniszyn et al. [1] is among the first to independently confirm the clinical observations from Frudinger et al. [5]. Romaniszyn et al.'s findings are particularly timely since Frudinger et al. [6] just published a follow-up to their original clinical cohort documenting persistent improvements in patient symptoms for up to 5 years [6].

Multipotent stromal/stem cells can be isolated from skeletal muscle, bone marrow, and adipose tissue [7]. Due to their relatively easy access in most adults, preclinical and clinical studies exploring the use of autologous and allogeneic cell-based therapies have increased exponentially over the past two decades [8, 9]. The US National Institutes of Health tracks the number of registered clinical trials, and their Web site (www.clinicaltrials.gov) offers a snap shot of the clinical landscape. A recent search of the Web site identifies 298 studies associated with "mesenchymal stem cells" and 159 associated with "skeletal muscle cells." These studies are conducted routinely by academia, government agencies, industry, and combinations thereof. In recent years, regulatory agencies such as the FDA and EMEA have substantially expanded their inhouse expertise for the quantitative assessment cell-based 
therapies [10]. Cell therapeutics need to be manufactured according to the strict guidelines of current Good Manufacturing Practices (cGMP) to insure that the biological products delivered to patients are free of bacterial, endotoxin, viral, or other contaminants, display a sufficient level of viability and functionality, and can be delivered safely without adverse events [9]. In the current study, the authors' culture expanded the skeletal-derived cells in medium supplemented with antibiotics and antimycotics. While they examined the myocyte differentiation and CD56 flow cytometry assays to confirm the identity of their cell product, no additional assays were reported to insure that the product was free of bacterial or endotoxin contamination [10]. Indeed, the presence of antibiotics and antimycotics would mask the detection of such contaminants during routine culture conditions. While it is fortunate that no adverse events associated with contamination were noted during the current study, contaminants are of particular concern in circumstances where cell therapies are administered to immunocompromised recipients.

The follow-up to this pilot study will require the accumulation and assessment of further experimental data. For example, it will be of critical importance to determine the dose dependency of the treatment outcomes. Questions remain: Is there an optimal number of cells to deliver per injection site and can you deliver too many or too few cells? Furthermore, the current study was conducted at a major university medical center; however, not all institutions will necessarily have access to cell culture facilities capable of expanding autologous cell products on site. This leads to additional questions: Can cells be manufactured at a centralized facility and returned to the clinician without loss of cell viability and with trackable safeguards such as bar-code labeling to insure that the patient's own autologous cells, rather than someone else's cells, are delivered back to her/him?

In summary, the current publication reflects the advancement of autologous cell therapy and the emergence of regenerative surgery as a discipline. Autologous cells are potential clinical tools, and these present exciting opportunities to improve treatment for fecal incontinence and other colorectal conditions, such as fistulas [11]. Practitioners should be prepared to incorporate this experimental technology once it has successfully navigated the regulatory hurdles documenting its safety and efficacy.

\section{Compliance with ethical standards}

Conflict of interest Dr. Gimble is the co-founder, co-owner, and Chief Scientific Officer of LaCell LLC, a for protit biotechnology company focusing on the development of research tools and processes to promote the clinical translation of stromal/stem cells.

Ethical approval All procedures performed in studies involving human participants were in accordance with the ethical standards of the institutional and/or national research committee and with the 1964 Helsinki declaration and its later amendments or comparable ethical standards.

Informed consent All patients provided informed consent prior to enrollment in the study.

\section{References}

1. Romaniszyn M, Rozwadowska N, Malcher A, Kolanowski T, Walega P, Kurpisz M (2015) Implantation of autologous musclederived stem cells in treatment of fecal incontinence: results of an experimental pilot study. Tech Coloproctol. doi:10.1007/s10151015-1351-0

2. Dominici M, Le Blanc K, Mueller I et al (2006) Minimal criteria for defining multipotent mesenchymal stromal cells. The International Society for Cellular Therapy position statement. Cytotherapy 8:315-317

3. Salcedo L, Mayorga M, Damaser M et al (2013) Mesenchymal stem cells can improve anal pressures after anal sphincter injury. Stem Cell Res 10:95-102

4. Salcedo L, Penn M, Damaser M, Balog B, Zutshi M (2014) Functional outcome after anal sphincter injury and treatment with mesenchymal stem cells. Stem Cells Transl Med 3:760-767

5. Frudinger A, Kolle D, Schwaiger W, Pfeifer J, Paede J, Halligan S (2010) Muscle-derived cell injection to treat anal incontinence due to obstetric trauma: pilot study with 1 year follow-up. Gut 59:55-61

6. Frudinger A, Pfeifer J, Paede J, Kolovetsiou-Kreiner V, Marksteiner R, Halligan S (2015) Autologous skeletal-muscle-derived cell injection for anal incontinence due to obstetric trauma: a 5 -year follow-up of an initial study of 10 patients. Colorectal Dis 17:794-801

7. Caplan AI (1991) Mesenchymal stem cells. J Orthop Res 9:641-650

8. Gimble JM, Guilak F, Bunnell BA (2010) Clinical and preclinical translation of cell-based therapies using adipose tissue-derived cells. Stem Cell Res Ther 1:19

9. Gimble JM, Bunnell BA, Chiu ES, Guilak F (2011) Concise review: adipose-derived stromal vascular fraction cells and stem cells: let's not get lost in translation. Stem Cells 29:749-754

10. Mendicino M, Bailey AM, Wonnacott K, Puri RK, Bauer SR (2014) MSC-based product characterization for clinical trials: an FDA perspective. Cell Stem Cell 14:141-145

11. Sanz-Baro R, Garcia-Arranz M, Guadalajara H, de la Quintana P, Herreros MD, Garcia-Olmo D (2015) First-in-Human Case Study: pregnancy in women with Crohn's perianal fistula treated with adipose-derived stem cells: a Safety Study. Stem Cells Transl Med 4:598-602 